\title{
Hadroproduction on nuclei: inclusive cross-sections and parametrizations.
}

\author{
M. Gostkin* (for the HARP-CDP group) \\ Joint Institute for Nuclear Research \\ E-mail: gostkin@nu.jinr.ru
}

Inclusive hadron production cross-sections of the interactions of few $\mathrm{GeV} / c$ protons and charged pions with nuclei are of interest for the understanding of the underlying physics, the modeling of Monte Carlo generators of hadron-nucleus collisions, and the design of neutrino beams. Precise and comprehensive double-differential inclusive hadron production cross-sections from $B e, C$, $\mathrm{Cu}, \mathrm{Ta}$ and $\mathrm{Pb}$ target nuclei are presented and their characteristics discussed, with emphasis on their dependence on the nuclear mass number.

35th International Conference of High Energy Physics - ICHEP2010,

July 22-28, 2010

Paris France

\footnotetext{
* Speaker.

†The members of the HARP-CDP group are: A. Bolshakova, I. Boyko, G. Chelkov, D. Dedovich, A. Elagin, D. Emelyanov, M. Gostkin, A. Guskov, Z. Kroumchtein, Yu. Nefedov, K. Nikolaev and A. Zhemchugov from the Joint Institute for Nuclear Research, Dubna, Russian Federation; F. Dydak and J. Wotschack from CERN, Geneva, Switzerland; A. De Min from the Politecnico di Milano and INFN, Sezione di Milano-Bicocca, Milan, Italy; V. Ammosov, V. Gapienko, V. Koreshev, A. Semak, Yu. Sviridov, E. Usenko and V. Zaets from the Institute of High Energy Physics, Protvino, Russian Federation.
} 

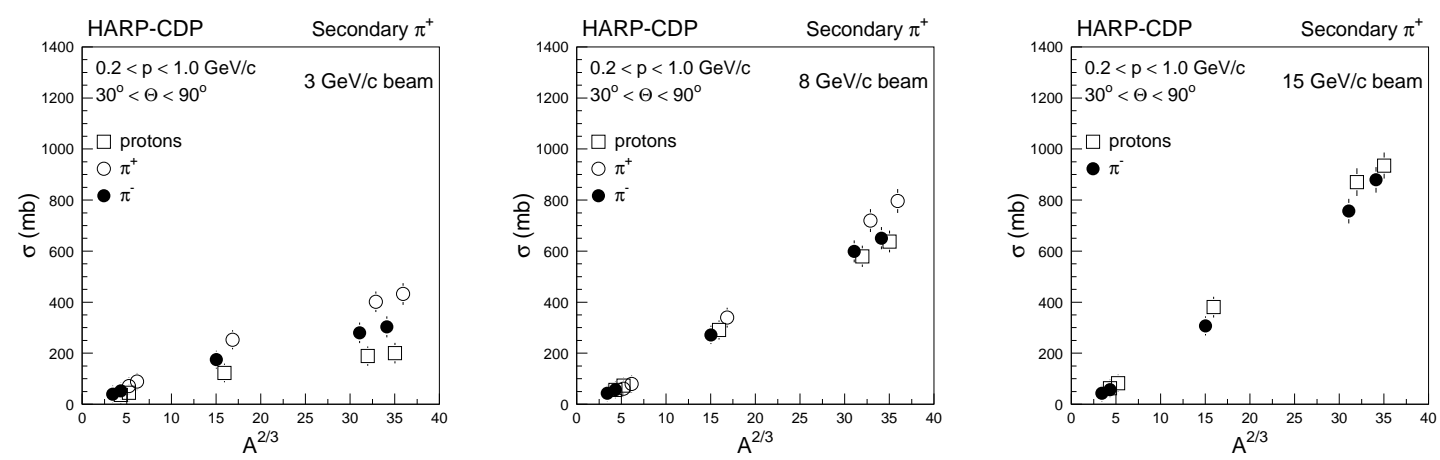

Figure 1: Inclusive cross-sections of $\pi^{+}$production by protons (open squares), $\pi^{+}$(open circles), and $\pi^{-}$ (black circles), as a function of $A^{2 / 3}$ for, from left to right, $B e, C, C u, T a$, and $P b$ nuclei.

The HARP experiment at CERN was carried out to measure inclusive cross-sections of the production of $p, \pi^{+}$and $\pi^{-}$, by $p, \pi^{+}$and $\pi^{-}$beams with momenta between 1.5 and $15 \mathrm{GeV} / c$, on target nuclei ranging from hydrogen to lead. The HARP-CDP group published so far inclusive cross-sections of $p, \pi^{+}$and $\pi^{-}$production on $B e, C, C u, T a$, and $P b$ nuclei (Refs. $[1,2,3,4,5,6]$ ).

Figure 1 presents a comparison between the inclusive cross-sections of $\pi^{+}$production, integrated over the secondaries' momentum range $0.2<p<1.0 \mathrm{GeV} / c$ and polar-angle range $30^{\circ}<$ $\theta<90^{\circ}$, in the interactions of $p, \pi^{+}$and $\pi^{-}$with $B e, C, C u, T a$ and $P b$ nuclei, for beam momenta of 3,8 and $15 \mathrm{GeV} / c$. The comparison employs the scaling variable $A^{2 / 3}$ where $A$ is the nuclear mass number of the respective nucleus. We note the approximately linear dependence on this scaling variable. At low beam momentum, the slope exhibits a strong dependence on beam particle type, which tends to disappear with higher beam momentum.

Figure 2 shows the increase of the inclusive cross-sections of $\pi^{+}$and $\pi^{-}$production by incoming protons of $8.0 \mathrm{GeV} / c$ (in the case of beryllium target nuclei: $+8.9 \mathrm{GeV} / c$ ) from the light beryllium nucleus to the heavy lead nucleus, for pions in the polar angle range $20^{\circ}<\theta<30^{\circ}$. It is interesting to note that $\pi^{-}$production is slightly favoured on heavy nuclei, while $\pi^{+}$production is slightly favoured on light nuclei.

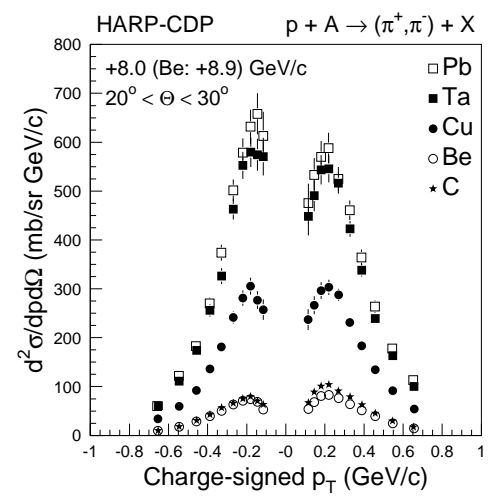

Figure 2: Inclusive pion production cross-sections.

\section{References}

[1] A. Bolshakova et al., Eur. Phys. J. C62 (2009) 293

[2] A. Bolshakova et al., Eur. Phys. J. C62 (2009) 697

[3] A. Bolshakova et al., Eur. Phys. J. C63 (2009) 549

[4] A. Bolshakova et al., Eur. Phys. J. C64 (2009) 181

[5] A. Bolshakova et al., Eur. Phys. J. C66 (2010) 57

[6] A. Bolshakova et al., accepted for publication in Eur. Phys. J. C, arXiv: 1007.5482 [hep-ex] 